\title{
All the physical world as mathematical: Physical entity as quantum information
}

\author{
Vasil Penchev, \\ Bulgarian Academy of Sciences: Institute of Philosophy and Sociology: \\ Dept. of Logical Systems and Models \\ vasildinev@gmail.com
}

Abstract: Quantum mechanics was reformulated as an information theory involving a generalized kind of information, namely quantum information, in the end of the last century. Quantum mechanics is the most fundamental physical theory referring to all claiming to be physical. Any physical entity turns out to be quantum information in the final analysis. A quantum bit is the unit of quantum information, and it is a generalization of the unit of classical information, a bit, as well as the quantum information itself is a generalization of classical information. Classical information refers to finite series or sets while quantum information, to infinite ones. Quantum information as well as classical information is a dimensionless quantity. Quantum information can be considered as a "bridge" between the mathematical and physical. The standard and common scientific epistemology grants the gap between the mathematical models and physical reality. The conception of truth as adequacy is what is able to transfer "over" that gap. One should explain how quantum information being a continuous transition between the physical and mathematical may refer to truth as adequacy and thus to the usual scientific epistemology and methodology. If it is the overall substance of anything claiming to be physical, one can question how different and dimensional physical quantities appear. Quantum information can be discussed as the counterpart of action. Quantum information is what is conserved, action is what is changed in virtue of the fundamental theorems of Emmy Noether (1918). The gap between mathematical models and physical reality, needing truth as adequacy to be overcome, is substituted by the openness of choice. That openness in turn can be interpreted as the openness of the present as a different concept of truth recollecting Heidegger's one as "unconcealment" $(\dot{\alpha} \lambda \dot{\eta} \theta \varepsilon 1 \alpha)$. Quantum information as what is conserved can be thought as the conservation of that openness.

Furthermore, the consideration of "all the physical world as mathematical" can be deduced from the "principle of constructive mathematizability of any theory" (@ EasyChair: "All Science as Rigorous Science: The Principle of Constructive Mathematizability of Ant Theory") under the following additional condition. The world is cognizable absolutely and thoroughly: a relevant theory for any entity can exist. Thus, the present paper can be considered as referring to the "ontological correlate" of "all rigorous science" meant in the former paper. Nonetheless, that deduction is not available here, but it is intended in the near future.

Key words: physical entity, quantum information, theorems of Noether (1918), unity of mathematics and physics, Heidegger's Aletheia, 


\section{Background and prehistory}

Quantum mechanics can be considered as an information theory. It was reformulated as an information theory involving a generalized kind of information, namely quantum information, in the end of the last century.

Furthermore, quantum mechanics is the most fundamental physical theory referring to all claiming to be physical. Thus, any physical entity turns out to be quantum information in the final analysis.

The quantum information being a generalized kind of information is omnipresent. That deduction can be reproduced very easily:

Any quantum state of any quantum system (what anything is) is a wave function, i.e. a point in the complex separable Hilbert space (which is the basic mathematical formalism of quantum mechanics). Then, any wave function can be represented as a series of quantum bits (qubits). Consequently, all in the universe are qubits, i.e. quantum information.

The concept of 'quantum bit' may be elucidated as follows:

A quantum bit is the unit of quantum information. It is a generalization of the unit of classical information, a bit, as well as the quantum information itself is a generalization of classical information. The concept of qubit is introduced by quantum mechanics as the normed superposition of two orthogonal subspaces of the separable complex Hilbert space. In turn, the separable complex Hilbert space is the structure underlying quantum mechanics.

A smooth transition exists from classical to quantum information. That transition is implied by the most fundamental equation in quantum mechanics, the Schrödinger equation, if it is reformulated in terms of quantum information. Then its "left side", referring to the partial time derivative of the wave function, means classical information in bits while its "right side" referring to the second "space" derivative of the wave function, quantum information in qubits (Penchev 2015).

The philosophical meaning of the generalization from classical to quantum information can be represented not less simply:

Classical information refers to finite series or sets while quantum information, to infinite ones. If a bit represents the choice between two equally probable alternatives, a qubit is the choice between an infinite set of alternatives. The definition of a qubit in quantum mechanics (suggested above) is different, but equivalent to the one suggested here.

\section{The problem}

Our questions are formulated on the background of quantum information:

How do physical dimensions appear?

Quantum information as well as classical information is a dimensionless quantity. If it is the overall substance of anything claiming to be physical, one can question how different and dimensional physical quantities appear both originating from it and reducible to it. Is quantum information conjugate to the physical quantity of action? 
If yes, one should interpret the fundamental theorems of Emmy Noether (1918) referring to action as well as to the ways it to be divided into two physical quantities.

The concept of 'dimension' is able to be a "bridge" between mathematics and physics. Nonetheless, quantum information can be considered as another "bridge" between the mathematical and physical.

The standard and common scientific epistemology, on the contrary, grants the gap between the mathematical models and physical reality. According to it, only the conception of truth as adequacy is what is able to transfer over that gap.

Then, one should be able to explain how quantum information being a continuous transition between the physical and mathematical may refer to truth as adequacy and thus to the usual scientific epistemology and methodology.

\section{Comments to the problem}

The meaning of Emmy Noether's theorems (1918) for physics at all, its philosophy, and particularly, for quantum mechanics is immense. The "focus" of our interpretation is shifted slightly, but intentionally. That "refocusing" may be described as follows: (a) What is meant as a universal physical quantity in the theorems at issue is action and only action. So, they privilege action as the most fundamental physical quantity, by which all others are underlain. That privileging is not invented by them, but it is rather extracted by the development of mechanics (including quantum mechanics), where the principle of least action is one of the most fundamental and heuristic ones and formulated explicitly in various and generalized forms a long time ago.

(b) They discuss any possible division of the action within any physical system into two parts independent of each other: the one is "what is changed"; the other one and its counterpart is "what is conserved".

(c) So, they state implicitly (or as their condition) that change and conservation in any physical system can be distinguished from each other disjunctively and furthermore described quantitatively independently of each other always. Action is that fundamental physical quantity able to unify those two metaphysical categories (namely change and conservation) as to all the physical.

(d) Only on that base sketched in "a-c", the explicit statements of both theorems can be elucidated in a generalized way: they reveal the link of the law of change by means of its "n" variables or "parameters" and the law of conservation by means of its " $n$ " differential equations to be extremely simple. Their number is the same, "n". Particularly, the first theorem investigates the "degenerated" case where all variables of change are constants, or properly "parameters", and the corresponding differential equations of conservation are in turn "degenerated" to the "n" times repeated tautology "the differential of any constant is zero" .

\footnotetext{
${ }^{1}$ One may notice, that the solution of the system of "differential equations" in that degenerate case can be directly interpreted as the variables of change, both being " $n$ " constants. One may admit that the interpretation of the solution of the equations as the variables of change might hold in the case of the second theorem. However, it does not state
} 
(e) If one involves the concept of 'symmetry' as the "constancy of change", the usual focus of interpretation centered on the first theorem may be restored secondarily in our viewpoint as the coincidence of the "constancy of change" with the "constancy of conservation". Thus, the shift of interpretation may be described as refocusing to both implicit ontological premises and philosophical meaning of the theorems after us.

(f) Meaning our intention further to involve '(quantum) information' as the counterpart of the physical action itself, still one similarity needs a certain formulation expressively. The formal structure of the disjunctive division of the action into two correlative counterparts of change and conservation correspondingly possesses the formal structure of a $\mathrm{bit}^{2}$ of information.

That background of how Noether's theorems are understood addresses directly the problem of the genesis of dimensions in a properly physical sense and furthermore linked to its mathematical counterpart. The two fundamental theorems should be involved for investigating the problem of dimensions both physical and mathematical:

The distinctiveness of change and conservation to be disjunctive from each other generates a fundamental gap, philosophical, physical, and mathematical. It underlies any split of physical action into possible pairs of quantities with complementary (or "conjugate") physical dimensionalities.

Furthermore (but in a different way), it underlies the philosophical distinctiveness of 'quantity' and 'quality' and the mathematical distinctiveness of 'variable' and 'values'. However, the two physical quantities of different physical dimensionality, to which action is decomposable, are also two different qualities and two different variables rather than a quantity and a quality, and rather than a variable and its values. The gap between their physical dimensions exists in virtue of the former rather than in virtue of the latter two ones.

Anyway, one may investigate the special case where the two "kinds" of gaps between dimensions, namely between 'quality' and 'quantity', or between 'variable' and 'values', on the one hand, and in virtue of Noether's theorems, on the other hand, would coincide to each other for both share the division between change and conservation.

That special case should obey the following condition. It should be a dimensionless physical quantity in virtue of Noether's theorem. That dimensionless physical quantity can be considered as values of its counterpart conjugate to action. This implies that its counterpart possesses the physical dimensionality of action. The simplest conjecture satisfying that condition is the physical action itself.

Resuming, Noether's theorem determine the links between the conserved quantities, e.g. energy, and the changeable, e.g. time, in any physical system. The product of the former and the latter has always the physical dimension of action (what the dimension of the fundamental Planck constant is) and thus, it can be interpreted as the physical quantity of action. One can consider the particular case where what is changed is physical action. If what is changed

this, but something much weaker, which is a trivial corollary from the much stronger coincidence of the variables of change and the variables-solution of the system of differential equations of conservation.

${ }^{2}$ It can be defined as the disjunctive choice between two equally probable alternatives, which may be "change" and "conservation". 
is physical action, the theorems of Emmy Noether imply that what is conserved should be dimensionless physically. Quantum information being namely dimensionless physically seems to be an admissible applicant for the counterpart of action.

Two examples, which will be used further in this text (in the description of the "bridge" between the Einstein field equation and the Schrödinger equation), may elucidate the meaning of the first Noether theorem to both action as what is changed and quantum information as what is conserved:

1. Global symmetry of action in quantum mechanics. If the functional of action is identical to the action itself as far as the interpretation of the action itself as what is changed requires in virtue of the first Noether theorem, this implies the symmetry of Hilbert space utilized in quantum mechanics and its dual counterpart identical to the former. That Hilbert space can be interpreted as the free variable of quantum information and any wave function as a certain value $e^{3}$ of it. Consequently, that global symmetry of action implies the conservation of quantum information as the Hilbert space of quantum mechanics is its universal mathematical formalism.

2. Global symmetry of action in general relativity. A main obstacle fortunately soluble exists for the approach to the global symmetry of action in quantum mechanics to be repeated as to general relativity: its pseudo-Riemannian space is "curved", and thus the corresponding dual counterparts (the covariant one and the contravariant one) are different. Its pseudo-metrics and finite dimensionality are not any trouble, for the imaginary (physically, subluminal) domain of Minkowski space being also pseudo-metrical and four-dimensional admits an isomorphism to the separable complex Hilbert space of quantum mechanics (Penchev 2013) under the additional condition of the axiom of choice ${ }^{4}$. So, the problem would be resolved if one builds a physically meaningful isomorphism between the real (physically, superluminal) domain and the imaginary (subluminal physically) domain of pseudo-Riemannian space (Penchev 2013) for a very simple symmetry exists between the real and imaginary domain of Minkowski space. Action is an internal quantity to those spaces physically interpreted ${ }^{5}$, and consequently that series of more than one isomorphism conserves any symmetry of action implying a global symmetry of action in general relativity very similar and even identical in essence to the analogical symmetry in quantum mechanics.

\section{The thesis}

The thesis on that background of the above comment to the problem refers to the conservation of quantum information:

Quantum information is conserved, being the counterpart of the changeable action.

\footnotetext{
${ }^{3}$ The variable of quantum information can be visualized as a quantum machine of Turing, i.e. as an infinite series of qubits; correspondingly, "free variable", as "empty" sells without any recording in each of them, and "wave function" as the well-ordered set of certain records in each of them.

${ }^{4}$ Involving the axiom of choice is justified intuitively as the "set-theory quantization" therefore referring to Skolem's "paradox" (or said better, "Skolem's argument").

${ }^{5}$ Action is defined as a variable of both dual counterparts within each space in question.
} 
One may notice an ostensible contradiction as to what is changed and what is conserved if action and quantum information are conjugate counterparts in the sense of Noether's theorem: From the viewpoint of "variable and its values" as in a few paragraphs ago, action is the variable or quality, after which quantum information is equivalent to its dimensionless values, for itself is dimensionless physically.

From the proper viewpoint of Noether's theorems as above, action is what is changeable, and quantum information is what is conserved. One may complement that the case of action conservation seems to be inacceptable after physical consideration as far as energy is its first time derivative though partial. This implies that the time contribution of action to energy would be totally zero though locally not in general. Also vice versa: energy does not cause any physical action globally, which seems to be contradictory (though only to our common sense maybe).

The above sense of Noether theorems may be restored even from a properly mathematical viewpoint for they mean the functional of action, and thus a variable ("A") of variables ("B") (i.e. a set of functions "B" mapped by means of " $A$ " into a set ("C $C$ ") of constants: " $A(B)=C$ "). Of course, it is a complex function and therefore it may be decomposed into two simple functions, namely " $\mathrm{A}(\mathrm{B})$ " and " $\mathrm{B}(\mathrm{C})$ ", however on two successive hierarchical levels where " $\mathrm{A}(\mathrm{B})$ " is situated on the meta-level, and " $\mathrm{B}(\mathrm{C})$ ", on the level itself (i.e. the level immediately lower than the "meta-level"). Then, the former ("meta-level") is "what is conserved" as a shared single quality or "free variable", and the latter ("level") is "what is changed" as its values. The second Noether theorem considers the general case where an additional mapping substitutes the identity link " $B=B$ " of the first one: namely, " $[\mathrm{B}(\mathrm{B})=\mathrm{B}$ "]" where " $\mathrm{A}(\mathrm{B})=\mathrm{A}(\mathrm{B}$ '), and " $\mathrm{B}(\mathrm{C})=\mathrm{B}$ " $(\mathrm{C})$ ". Thus, the first theorem considers the case of two neighboring hierarchical levels, and the second one, of two arbitrary hierarchical levels therefore linked to each other by " $[\mathrm{B}(\mathrm{B}$ ') $=\mathrm{B}$ "]" $]$. At last, one may admit still one symmetry as an additional option: the change of the direction of hierarchy and thus, of the ordering of the levels.

One may suggest further that the universe where action is changeable, and quantum information is conserved, on the one hand, and the "reverse" universe where action is conserved, and quantum information is variable, on the other hand, is one and the same in the final analysis. In a sense justified above, Noether's theorem equate change and conservation, and concept of action serves for, or may be defined right under condition of that unification.

Furthermore, that same universe splitting itself in two possible and disjunctive ways into a "mathematical one" with changeable information, but constant action globally, and a "physical one" (vice versa) share again (or maybe tautologically) the fundamental structure of a bit of information just as the physical action is split of change and conservation in virtue of Noether's theorems.

Anyway, the further discussion is only within the framework of the physical universe of changeable action and quantum information conservation.

If the change of action is uniform in time, energy is conserved. However, the conservation of quantum information is more universally than energy conservation for if the action is changed (i.e.

\footnotetext{
${ }^{6}$ An unexpected connection to Russell's theory of types and thus, to the foundation of mathematics may be found.
} 
not only uniformly), its counterpart of quantum information is conserved. Energy conservation implies quantum information conservation, but not vice versa.

One existing and exceptionally elaborated example for that generalized approach to conservation is general relativity. Energy may be transformed in momentum in it immediately because of the "curvature" of space-time in it according to its most fundamental equation: the Einstein field equation.

The factual link between general relativity and Noether's theorems is essential and very instructive for their philosophical meaning and understanding. It arose in the course of the debate of Hilbert and Einstein (Mehra 1974) about the causality violation in the latter's theory according to the former (Renn, Stachel 2007) as early as 1915-1916 (Hilbert 1924) ${ }^{7}$ as well as about the problem of reality.

That discussion is centered about Einstein's "argument about hole" suggested by himself ${ }^{8}$ as an heuristic counterargument for the further elucidation of the principle of general covariance ${ }^{9}$ in theory of relativity. Einstein (1920: 106-108) generalized as the problem as its eventual solution to how one should understand concept of space (space-time) in the framework of general relativity. John Norton synthesized its essence so: "if the field equations are generally covariant, then a given stress-energy tensor cannot uniquely determine the gravitational field through the field equations" (Norton 1984: 286).

The initial solution of Einstein insisted of space-time to be secondary for one may measure it only by means of space-time coincidences with devices having mass ${ }^{10}$ (Einstein 1916: 776). Thus,

\footnotetext{
7 The approach of Hilbert to gravitation is different from that of Einstein in many aspects (Mehra 1974). The starting point from the principle of least action rather than that of general covariance is the most essential in the context of Noether's theorems. The former seems to imply the problem of causality violation right because of Einstein's hole rgument being developed further by Hilbert as a refuting counterargument to general relativity underlain by general covariance. However, a not less dangerous problem, namely the violation of energy conservation seemed to be implied by Hilbert's approach. He discussed it in the correspondence with F. Klein (Klein 1917; Hilbert, Klein 1985) as well as the viewpoint of Emmy Noether. These three great scientist are connected by close life and scientific relationships (Rowe 1999). "The Inception of the Noether Theorems" (Kosmann-Schwarzbach 2011: 29-53) can be revealed in the sketched historical context. However, one may demonstrate (as us further in the main text) the philosophical fundamentality of them as reconciling Einstein's approach to gravitation by general covariance and Hilbert's one by least action and thus, a "bridge" between general relativity and quantum mechanics.

${ }^{8}$ The initial mentions (1913-1914) in works of Einstein and Grossmann (or Einstein alone) are enumerated in Footnote 44 (Norton 1984: 284) re-referring to other footnotes in the same paper.

${ }^{9}$ Einstein has formulated the principle many times in different papers with slight or contextual differences. We use this one for it is in the present context of the "hole argument" or "Relativität und Raumproblem" (Einstein 1920: 91109): "Die allgemeinen Naturgesetze sind durch Gleichungen auszudrücken, die für alle Koordinatensysteme gelten, d. h. die beliebigen Substitutionen gegenüber kovariant (allgemein kovariant) sind" (Einstein 1916: 776). "The general laws of nature are to be expressed by equations which hold for all systems of co-ordinates, that is, are covariant with respect to any substitutions whatever (generally covariant)" (cited according to the English translation by W. Perrett and G.B.Jeffery in "The Principle of Relativity. A collection of original memoires on the special and general relativity by H.A. Lorentz, A. Einstein, H. Minkowski and H. Weyl (A. Sommerfeld, ed.). New York: Dover publications, 1952, p. 117.)

${ }^{10}$ One may notice that the secondarity of space-time to mass-energy implies "Mach's principle" (Einstein 1918) also consistent to the "cosmological constant" in the same paper not to be allowed of the universe to extend, and called by Einstein himself his "biggest blunt" (Gamov 1970: 44).
} 
any all possible space-time metrics are reduced to a single one after the availability of a mass distribution or ... after measurement if one emphasizes the connection to quantum mechanics.

He was rather more cautious in a later work (Einstein 1920: 108) maybe influenced or inspired by the paper of Emmy Noether (1918) meanwhile. He distinguished the space-time of general relativity from Minkowski space (of special relativity) by the unavailability/ availability of a global symmetry in Footnote 29 as a direction of a rigorous solution obtained by him.

Anyway, that symmetry (maybe the first gauge symmetry in a fundamental physical theory) is valid locally for the pseudo-Riemannian space of general relativity right in virtue of the second theorem of Noether (Klein 1918; 1918a).

The way for the principle of general covariance (Einstein) and that of least action (Hilbert) to be reconciled in general relativity needs the second theorem of Emmy Noether:

If one considers general covariance as a complex gauge symmetry parametrized by a single function, the theorem implies for one differential equation of the Lagrangian to exist. The Einstein field equation has to be that equation for it is deduced just by general covariance. Its solution privileges reference frames as the real ones. As for as the consideration here is only qualitative, one may grant that the privileged reference frame is a single one for the simplicity of explanation.

As it is explained above, both Noether theorems distinguishes what is changed (reference frames according to general covariance in the case) from what is conserved (the solution of the Einstein field equation) disjunctively. This means that they are absolutely independent of each other, and consequently the privileging of a reference frame as the real one is absolutely random as one should expect according to general covariance.

The classical principle of least action cannot hold for energy conservation is a direct corollary from it, and energy conservation is not valid as to general relativity in certain cases. Anyway, it holds in a generalized sense, namely as that absolutely independence of change and conservation as above and thus confirming again general covariance by the absolutely random choice of one reference frame as the real one.

The bridge of gauge invariance built by the second theorem can be repeated as to the Schrödinger equation and the joint gauge symmetry $\{[\mathrm{U}(1)] \mathrm{X}[\mathrm{SU}(2)] \mathrm{X}[\mathrm{SU}(3)]\}$ of the Standard model in a reverse order to that after general relativity:

Know we what is conserved, namely the solution of the Schrödinger equation, the theorem implies for a certain gauge symmetry to exist. All experiments confirmed and continue to confirm that it should be that of the Standard model.

Thus, the second theorem allows of a far reaching analogy between the Einstein field equation and the Schrödinger one and thus, between general relativity and quantum mechanics. A few questions appear immediately:

How far reaching? Even to an isomorphism under an additional condition? What condition and would it be consistent to both general relativity and quantum mechanics? If yes, whether it is deductible from one of them or from both or independent? What might the physical meaning of that condition be? 
A few main and huge obstacles may be enumerated on the way from an analogy to an isomorphism under certain conditions:

(a) How to proportionate the fundamental physical constants such as the light speed in vacuum, "c", the gravitational constant, "G", and the Planck constant, " $h$ ", for "G" is not available in the Schrödinger equation, and " $h$ " not in the Einstein field equation?

(b) How to harmonize the pseudo-Riemannian space of general relativity with the separable complex Hilbert space for the former is four-dimensional, "curved", and pseudo-metric, and the latter is infinite-dimensional, "straight", and metric? Particularly, how to transform a world line being an infinite well-ordered subset of points of the former into a certain wave function being a point of the later, and vice versa, too?

(c) How to suit the general covariance as the fundamental gauge symmetry of general relativity to the joint gauge symmetry $\{[\mathrm{U}(1)] \mathrm{X}[\mathrm{SU}(2)] \mathrm{X}[\mathrm{SU}(3)]\}$ of the Standard model? Particularly, how to interpret the concept of reference frame in terms of the Standard model?

(d) How to unify the Schrödinger equation and the Einstein field equation for they are absolutely different kinds of differential equations?

(e) How to reconcile the continuity (even smoothness) of general relativity with the discreteness of quantum mechanics, on the one hand, and the actuality of the former with the Born probabilistic interpretation of the latter, on the other hand, as well as both aspects (on both "hands") to each other?

(f) How to cohere the physical interpretation of both fundamental and very well confirmed theories for general relativity refers to megascopic astronomical objects measured by macroscopic devices, and quantum mechanics, to microscopic entities measured by macroscopic apparatuses?

Though the obstacles seem to be overpowering, one may ask whether the Noether theorems imply and thus assure at least the existence of that isomorphism under certain additional conditions. If one proves the isomorphism of "what is changed" (i.e. the corresponding gauge symmetries) under certain additional conditions, does this imply the isomorphism of "what is conserved" (i.e. the corresponding differential equations) under the same conditions?

Those additional condition may be thought as the "obstacle (c)" above: in other words, is the gauge symmetry $\{[\mathrm{U}(1)] \mathrm{X}[\mathrm{SU}(2)] \mathrm{X}[\mathrm{SU}(3)]\}$, the quantized counterpart of general covariance? This "shifts the focus" for gravity to be quantized from the interaction itself (gravitation) to its condition according general relativity, namely general covariance by means of the above conjecture linked to the Noether theorems.

The conjecture may have been proved already or not yet. Anyway, it seems to be convincing and intuitively believable in a philosophical viewpoint demonstrated in the present paper. Indeed, the condition for the identity of "what is changed" to imply the identity of "what is conserved" may be a global symmetry of action generalizing the principle of least action as to gauge symmetries. It implies the conservation of quantum information as the conjunctive physical counterpart of action according to the first Noether theorem. 
If one continues in the philosophical pathway of interpretation and the conservation of quantum information is thought as the permanence of "openness" (or "unconcealment"), just this is the philosophical sense of the condition for the conjecture.

As to the quantization itself of general covariance, one may add still one consideration "pro". Any reference frame needs three relative quantities to be determined exhaustively: position, velocity, and acceleration. Their changes are linked to each other unambiguously as the first and second time derivative of the position change. However, each of them should be an independent quantity and thus symmetry after quantization, and therefore, be an independent condition of a corresponding interaction such as the electromagnetic, weak, and strong interactions separately, to which gravity might be decomposed eventually after quantization. One may conjecture about the parallelism of the following pairs after the quantization of general covariance: position and U(1); velocity and SU(2); acceleration and SU(3) (Penchev 2014).

\section{Philosophical corollaries}

Still a few interesting and philosophically meaningful corollaries are implied by the thesis: 1. Quantum information can be considered as the ultimate substance of the world. In other words, quantum information is the real substance of the world for it is conserved always (Penchev 2016). That conclusion is consistent to the interpretation of any wave function as a value of quantum information and thus as the universal physical substance of the world.

That conservation can be described further philosophically as the equivalence of possibility and actuality and thus as necessity in many ways, e.g. as follows. Any wave function can be considered as the characteristic function of a certain probability distribution. That probability distribution can be restored absolutely (in principle) in a long enough series of measurements of the entity, the states of which the wave function at issue describes exhaustively. The same procedure can be interpreted as a consequence of absolutely random choices assured by the axiom of choice and therefore, able to transform the coherent state of all possibilities into an equivalent well-ordered ${ }^{11}$ series of actual choices.

The identity of possibility and actuality defines necessity in a philosophical sense ${ }^{12}$. If the above equivalence is granted as the philosophical identity of possibility and actuality and thus implying necessity, the next step is that necessity to be interpreted in turn as the necessity for quantum information to be conserved.

Thus, quantum information might contain an internal (mathematical) proof for its necessity to be conserved.

In fact, the fundamental theorems about the absence of hidden variables in quantum mechanics (Neumann 1932: 167-173; Kochen, Specker 1968) and thus its "completeness" 13 can be interpreted as that internal proof of necessity for quantum information to be conserved since they deduce that absence only on the base of the mathematical formalism of the separable complex Hilbert space.

\footnotetext{
${ }^{11}$ In the rigorous mathematical meaning of "well-ordering".

12 For example, as this is discussed in Koons, Pickavance 2017.

${ }^{13}$ Versus the conjecture of its alleged "incompleteness" (Einstein, Podolsky, Rosen 1935).
} 
Indeed, the availability of hidden variables, or Einstein, Podolsky, and Rosen's "incompleteness", would mean the option for quantum information to be changed.

2 Quantum information and action are linked to each other intimately. What is changed, namely physical action, appears necessarily in virtue of Emmy Noether's theorems as the counterpart of quantum information once it is conserved always, i.e. universally. Thus, if what is conserved necessarily is interpreted as the ultimate substance of the world, its counterpart, namely, action, is what is changed universally. Action can be thought as the change itself at all as well as the philosophical concept of 'being' different from substance, but correlated to it even mathematically and physically.

3. The "no Creator" hypothesis seems to be shared by science and almost all branches of. Maybe it implies the "no Big Bang" hypothesis for there exist two different, and maybe even inconsistent conjectures in physics about the "creation":

The "Big Bang" is the most popular one. It postulates a special point of the creation (the "singularity" in " $t=0 "$ ), in which physical laws do not hold yet. However, they (first of all, both energy conservation and reversible time) hold at any time thereafter.

The viewpoint of quantum mechanics is different and rather similar to that of Descartes: mainly in the "Third Meditation" (Descartes 1641; Husserl 1931; Secada 1990; Gorham 2007, 2008):

The creation is permanent, at any time, and it is due to the irrevrsibility of time. If one projects all irreversibility of time into a single point of the beginning, the well-known picture of the "Big Bang" would appear.

One can search for empirical confirmations versus the "Big Bang". If the "Big Bang" was real, any physical objects in the universe older than the universe itself would be impossible to exist. However, if the "Big Bang" is not real, but only a hypothetical projection of the irreversibility of time into a single zero point, it may be an averaging of the course of time in all points in the universe. Arbitrary deviations to that average quantity would exist in various spots of the universe. The most objects might be younger than the universe, but at least a few ones should be older than it. The existence of objects older than the universe is partly ${ }^{14}$ confirmed experimentally (Chamberlain, Aller 1951; Spite, Spite 1982; Molaro 1987; Bond et al. 2013). The opposite conjecture of the "Big Bang" was elaborated by the Belgian Catholic priest Georges Lemaître (1927; 1931; 1946), so early as in 1927. It was able to reconcile science (from the "Big Bang" on) with religion (the "Big Bang" itself as God's creation).

The philosophical essence of the "no Big Bang" hypothesis is: the creation is permanent and due to the irreversibility of time. The creation is mathematically necessary. Thus, it is not due to one's free will (e.g. God's, the observer's, etc.).

Generalizing philosophically, the being (or at least the physical being) appears necessarily in virtue of mathematical laws rather than randomly therefore and particularly not needing any

\footnotetext{
14 The contemporary accuracy of measurements do not allow for any unambiguous statement: they may or may not be older the universe really. However, the dominating paradigm of the "real Big Bang" influences very strongly onto the formulations: so, all publications emphasize that those objects have appeared very soon after the Big Bang rather than a little before it though the experimental accuracy allows of both kinds of interpretation.
} 
"creator" or other "ultimate cause" to be. On the contrary, the philosophical sense of the Big Bang is that of the "ultimate cause" accessible by the contemporary science.

Furthermore, time is thought in two opposite ways, inconsistent to each other, in different physical theories: either reversible (classical mechanics, relativity, etc.), or irreversible (thermodynamics, etc.).

The solution of statistical thermodynamics about time is very interesting and successful. It manages to resolve the contradiction of reversible time in mechanics and irreversible time in thermodynamics. The thermodynamic irreversibility according to it is a result of the statistical averaging of mechanical reversibility. A huge part of information is lost after averaging, and just that loss generates irreversibility in thermodynamics. The loss of information in thermodynamics can be generalized by the notion "hidden variables", hidden by, and after averaging. Quantum mechanics is that domain of physics which generates new fundamental questions about the relevant way how to be reconciled both reversible and irreversible time unto a single consistent scientific theory.

Quantum mechanics was forced to introduce the Planck constant, which is thermodynamic in essence, as fundamental to mechanical motion. Thus, it should reconcile the reversible time of mechanics with the irreversible time of thermodynamics already in its foundation. Many scientists, even Einstein, expected that its solution should be similar to that of statistical thermodynamics. However, that conjecture turned out to be fundamentally wrong: "No hidden variables in quantum mechanics!" might be the "slogan" of the solution about the reversible and irreversible time in quantum mechanics. Anyway, the solution of quantum mechanics is partly analogical to that of statistical thermodynamics. A huge part (exactly the half) of information is lost in any single measurement ${ }^{15}$. However, that loss is not due to averaging or to human ignorance. It is a fundamental law of nature due to the limitation imposed by the Planck constant. That fundamental loss is caused by the course of time in the final analysis. All rejected counterfactual alternatives might represent that necessary loss of information eventually.

All irreversibility of time is concentrated in a single point, namely the "Big Bang", after which the physical quantity of time is absolutely reversible as general relativity needs. However, time is irreversible in thermodynamics and ... according to our experience. Consequently, the concept of

\footnotetext{
${ }^{15}$ In fact, any quantum leap is determined unambiguously by both initial and final state. So, the number of necessary variables is exactly the same as the classical case of smooth motion, and not the half of it: only as kinds of variables are the half of them, but each of them twice: once for the leap initial state, and once more for the leap final state. However and unlike the classical case, that exhausting number of variables is not accessible in any single measurement, but in two ones eventually. Thus, a new problem appears as far as Heraclitus' "No man ever steps in the same river twice, for it's not the same river and he's not the same man" holds. Continuing the metaphor of Heraclitus" "river", quantum mechanics is forced to describe all states of both "river" (i.e. the investigated quantum entity) and "man" (i.e. the apparatus eventually together with the experimenter), which might happen in future: properly, this is the wave function of the entity at issue. The wave function refers only to the half variables in comparison to the classical case, but the information about them is doubled for wave function is complex rather than real. From that viewpoint, hidden variables in quantum mechanics cannot exist for the information is exactly the same as in the classical case. The half of information is lost only after measurement and then secondarily restorable as a probability distribution of all states of both "man" and "river" in a series of measurements.
} 
the "Big Bang" is possible to be an artefact (and thus a kind of correction) as far as the reversible time is utilized as universal in a huge part of physical science.

4. What is changed can be discussed versus what is conserved both mathematically and physically as Emmy Noether's theorems state, on the one hand, and philosophically, on the other hand. Quantum information and action are the same seen from two disjunctive viewpoints correspondingly as what is conserved and as what is changed. Thus, they do not need the concept of truth as adequacy necessary to link them over the gap for they are the same by themselves. If one sees both what is changed and what is conserved as the same in a fundamental ontological sense as the "being" in Heidegger's fundamental ontology, the corresponding concept of truth would be right the "unconcealment" (Heidegger's $\dot{\alpha} \lambda \dot{\eta} \theta \varepsilon 1 \alpha)^{16}$. The main feature of Heidegger's $\dot{\alpha} \lambda \hat{\eta} \theta \varepsilon 1 \alpha$ in our context is permanent openness as the conservation of the ability of change forever.

\section{A few arguments for the thesis briefly}

They are rather an explicit synopsis of the whole previous exhibition in the text seen now from the viewpoint of an extended or implicit argumentation of the thesis or as a discussion of the possible solution of the formulated problem:

1. Quantum information being dimensionless satisfies the necessary condition to be the dimensionless counterpart of action. Noether's theorems imply only that the counterpart of action has to be dimensionless as a physical quantity. Thus, it is only a necessary, but not sufficient condition: quantum information is not more than a possible applicant for it satisfies a necessary condition. However, the contemporary science cannot suggest any other applicant fundamentally different from it.

There is even an internal mathematical cause for the above statement. Quantum information can be proved internally, i.e. mathematically as necessary. Then any other applicant is equivalent to quantum information for that necessity.

2. Quantum mechanics for the omnipresent concept of quantum information can be discussed as "information mechanics". The fundamentality of quantum mechanics reformulated successfully in terms of quantum information is an argument for the thesis, too: The historical base for this reformulation in the end of 20th century and the beginning of 21 th century (e.g. Timpson 2013) is Max Born's probabilistic interpretation of quantum mechanics in the late twenties of XX century (Born 1926; 1927; 1954). The sense of that interpretation is the equivalence of any probability distribution of quantum states to a single actual quantum state. Further, the relation of quantum probability distributions is interpreted ${ }^{17}$ as information (Penchev 2015a, Penchev 2016a) according to the definition of information. Then, quantum mechanics is absolutely in the framework of a maybe more general theory of quantum information, discussing only the physical aspects of the latter as action in general.

3. Quantum information is defined naturally as a choice equivalent to a number of bits:

\footnotetext{
${ }^{16}$ A detailed bibliography about "Heidegger on $\dot{\alpha} \lambda \dot{\eta} \theta \varepsilon \iota \alpha$ " is: https://www.ontology.co/biblio/heidegger-aletheiabiblio.htm (accessed 6 September 2018).

${ }^{17}$ For example, as in the definition of information in Kolmogorov (1968).
} 
The fundamental Planck constant having the physical dimension of action allows for any physical action to be juxtaposed a natural number. That natural number can be interpreted as a number of bits of information. Quantum information by means of quantum bits can be interpreted as the choice of a certain number of bits among all natural numbers. Thus, if a bit can be juxtaposed to a single natural number, a quantum bit corresponds to a certain choice of any natural number.

4. Quantum information can be interpreted philosophically as "openness":

Quantum information meaning the number of choices among an infinite set of alternatives can be discussed as the conservation of openness for choice as the necessary condition of any physical change. That openness can be further identified as the openness of the present or in other words, as the availability of the present always as that, in which any physical change can occur. In turn, that fundamental openness for choice can be seen as "unconcealment", i.e. as Heidegger's $\dot{\alpha} \lambda \dot{\eta} \theta \varepsilon 1 \alpha$, a kind of the most fundamental concept of truth generalizing that of adequacy.

\section{Brief conclusions:}

1. Quantum information can be discussed as the counterpart of action.

2. Quantum information is what is conserved, action is what is changed.

3. The gap between mathematical models and physical reality, needing truth as adequacy to be overcome, is substituted by the openness of choice.

4. That openness in turn can be interpreted as the openness of the present as a different concept of truth recollecting Heidegger's one, $\dot{\alpha} \lambda \hat{\eta} \theta \varepsilon 1 \alpha$ as "unconcealment".

5. Quantum information as what is conserved can be thought philosophically as the conservation of that openness. 


\section{References:}

Bond, H. E. et al. (2013) "HD 140283: A Star in the Solar Neighborhood that Formed Shortly After the Big Bang," The Astrophysical Journal Letters 765 (1): L12.

Born, M. (1926) "Zur Quantenmechanik der Stoßvorgänge. Zeitschrift für Physik," 37 (12): 863-867.

Born, M. (1927) "Physical aspects of quantum mechanics," Nature 119 (2992), 354-357.

Born, M. (1954) "The statistical interpretation of quantum mechanics (Max Born - Nobel Lecture. NobelPrize.org. Nobel Media AB 2018. Thu. 6 Sep 2018),"

$<$ https://www.nobelprize.org/prizes/physics/1954/born/lecture/>

Chamberlain, J. W., Aller, L. H. (1951) "The Atmospheres of A-Type Subdwarfs and 95 Leonis," Astrophysical Journal 114 (1), 52-72.

Descartes, R. (1641) Meditations on First Philosophy with Selections from the Objections and Replies (transl. M. Moriarty). "Oxford World's Classics”, Oxford, University Press, 2008.

Gamow, G. (1970) My world line: an informal autobiography, New York, Viking Press

Einstein, A. (1916) "Die Grundlage der allgemeinen Relativitätstheorie," Annalen der Physik 49 (7): 769-822.

Einstein, A. (1918) "Prinzipielles zur allgemeinen Relativitätstheorie," Annalen der Physik 55 (4): 241-244.

Einstein, A. Podolsky, B., Rosen, N. (1935) "Can Quantum-Mechanical Description of Physical Reality Be Considered Complete?" Physical Review 47 (10): 777-780.

Einstein, A. (1920) Über die spezielle und die allgemeine Relativitätstheorie, Berlin, Heidelberg: Springer, 2009 ("Relativität und Raumproblem", pp. 91-109).

Gorham, G. (2007) "Descartes on Time and Duration," Early Science and Medicine 12 (1): 28-54.

Gorham, G. (2008) “Descartes on God's Relation to Time," Religious Studies 44 (4): 413-431.

Hilbert, D. (1924) "Die Grundlagen der Physik," Mathematische Annalen 92 (1-2): 1-32.

Hilbert, D., Klein, F. (1985) "Der Briefwechsel David Hilbert-Felix Klein (1886-1918)," in (G. Frei, ed.) Arbeiten aus der Niedersächsischen Staats- und Universitätsbibliothek Göttingen, vol. 19. Göttingen: Vandenhoeck \& Ruprecht, 1985.

Husserl, E. (1931) Cartesianische Meditationen und Pariser Vorträge, Den Haag, Nijhoff, 1950.

Kochen, S., Specker, E. (1968) "The problem of hidden variables in quantum mechanics," Journal of Mathematics and Mechanics 17 (1): 59-87.

Kolmogorov, A. (1968) "Logical basis for information theory and probability theory," IEEE Transactions on Information Theory 14 (5): 662-664.

Koons, R.C., Pickavance, T.H. (2017) The atlas of reality: a comprehensive guide to metaphysics, West Sussex, Wiley Blackwell ("Possibility, Necessity, and Actuality: Concretism", pp. 317-331).

Kosmann-Schwarzbach, Y. (2011) The Noether Theorems. Invariance and Conservation Laws in the Twentieth Century, New York - Dordrecht - Heidelberg - London, Springer.

Molaro, P. (1987) "Upper limit to the boron abundance in the population II star HD 1402," Astronomy and Astrophysics 183 (2), 241-246.

Klein, F. (1917) "Zu Hilberts erster Note über die Grundlagen der Physik," Nachrichten von der Gesellschaft der Wissenschaften zu Göttingen, Mathematisch-Physikalische Klasse 1917, pp. 469-482.

Klein, F. (1918) "Über die Differentialgesetze für die Erhaltung von Impuls und Energie in der Einsteinschen Gravitationstheorie," Göttinger Nachrichten 1918, pp. 171-189.

Klein, F. (1918a) "Über die Integralform der Erhaltungssätz und die Theorie der räumlichgeschlossenen Welt," Göttinger Nachrichten 1918, pp. 394-423.

Lemaître, G. (1927) "Un Univers homogène de masse constante et de rayon croissant, rendant compte de la vitesse radiale des nébuleuses extra-galactiques," Annales de la Société scientifique de Bruxelles, Sèrie A 47: 49-59.

Lemaître, G. (1946) L'Hypothèse de l'Atome Primitif: Essai de cosmogonie, Neuchâtel, Griffon; Paris: Dunod. 
Lemaître, G. (1931) The Beginning of the World from the Point of View of Quantum Theory, London, MacMillan.

Mehra, J. (1974) Einstein, Hilbert, and the theory of gravitation: historical origins of general relativity theory, Dordrecht - Boston, Reidel, 1974.

Neumann, J. von (1932) Mathematische Grundlagen der Quantenmechanik, Berlin, Springer (IV.2, pp. 167-173).

Noether, E. (1918) "Invariante Variationsprobleme," Nachrichten von der Gesellschaft der Wissenschaften zu Göttingen, Mathematisch-Physikalische Klasse 1918, pp. 235-257.

Norton, J. (1984) "How Einstein Found His Field Equations: 1912-1915," Historical Studies in the Physical Sciences 14 (2): 253-316.

Penchev, V. (2013) "The Kochen-Specker theorem in quantum mechanics: a philosophical comment," Philosophical alternatives 22 (1): 67-77; 22 (3): 74-83.

Penchev, V. (2014) "Is Mass at Rest One and the Same? A Philosophical Comment: on the Quantum Information Theory of Mass in General Relativity and the Standard Model," Journal of Siberian Federal University. Humanities \& Social Sciences 7 (4), 704-720.

Penchev, V. (2015) "Quantum information as the information of infinite series," https://easychair.org/my/preprint version?version=13027

Penchev, V. (2015a) "God's Dice," Actas: VIII Conference of the Spanish Society for Logic, Methodology, and Philosophy of Sciences (J. Martínez, García-Carpintero, J. Díez, S. Oms, eds.), Barcelona: Universitat de Barcelona, 2015, pp. 297-303.

Penchev, V. (2016) "Matter as Information. Quantum Information as Matter," Nodi. Collana di Storia della filosofia 2016 (2): 127-138.

Penchev, V. (2016a) "A Formal Model of Metaphor in Frame Semantics," Proceedings of the 41st Annual Convention of the Society for the Study of Artificial Intelligence and the Simulation of Behaviour. New York: Curran Associates, 2016, pp. 187-194.

Renn, J., Stachel, J. (2007) "Hilbert's Foundation of Physics: From a Theory of Everything to a Constituent of General Relativity," in Gravitation in the twilight of classical physics. The promise of mathematics (J. Renn, M. Schemmel, eds.). The Genesis of General Relativity (J. Renn, ed.), vol. 4. Springer, Dordrecht, pp. 857-973.

Rowe, D. E. (1999) “The Göttingen response to general relativity and Emmy Noether's theorems," in: The Symbolic Universe, Geometry and Physics 1890-1930 (J. J. Gray, ed.). Oxford - New York, Oxford University Press, 1999, pp. 189-233.

Secada, J. E. K. (1990) "Descartes on Time and Causality," The Philosophical Review 99 (1): 45-72.

Spite, F., Spite, M. (1982) "Abundance of lithium in unevolved halo stars and old disk stars Interpretation and consequences," Astronomy \& Astrophysics 115 (2): 357-366.

Timpson, C. G. (2013) Quantum Information Theory and the Foundations of Quantum mechanics, Oxford, Clarendon Press. 